\title{
Das SMB ist tot, es lebe das SMB!
}

\author{
Hans Stalder \\ Prof. Dr. med., Mitglied der Redaktion
}

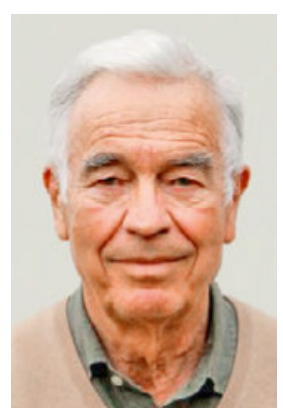

Der rasante Fortschritt in Technik und Therapie bedingt unabhängige Strukturen, die dem Arzt bei der Bewertung von Richtlinien und den Entscheidungsträgern im Gesundheitssystem bei der Beurteilung des wirtschaftlichen Mehrwerts helfen, die Richtigkeit ihres Handelns zu beurteilen. Seit Ende des letzten Jahrhunderts entstanden entsprechende Institutionen in verschiedenen Ländern. Die am besten bekannte ist das National Institute for Health and Care Excellence (NICE) in Grossbritannien. Vergleichbares gibt es in Frankreich (Haute Autorité de Santé, HAS), Deutschland (Institut für Qualität und Wirtschaftlichkeit im Gesundheitswesen, IQWiG) und andernorts in Europa. Alle diese Einrichtungen haben sich im European Network for Health Technology Assessment (EUnetHTA) zusammengeschlossen. Sie zeichnen sich aus durch ihre wissenschaftliche Kompetenz, ihre Transparenz und ihre Unabhängigkeit (sowohl von der Industrie, den Krankenkassen wie auch den medizinischen Leistungserbringern). Ihre Handlung besteht gemäss internationalen Standards in Begutachtung (Assessment) oder Health Technology Assessment (HTA), Bewertung (Appraisal) und Entscheidung. Bei der Begutachtung werden die verfügbaren wissenschaftlichen Nachweise einer systematischen Prüfung unterzogen, vor allem mit Blick auf Sicherheit und Zweckmässigkeit, aber auch in Bezug auf einen zusätzlichen Nutzen im Vergleich zu vorhandenen Alternativen. Bei der Bewertung werden juristische, ethische und soziale Aspekte miteinbezogen und - nach entsprechender Rücksprache mit den beteiligten Parteien (Ärzten, Industrie, Krankenkassen, Patienten ...) - Empfehlungen formuliert. Begutachtung und Bewertung müssen unabhängig vom Entscheidungsträger erfolgen.

In der Schweiz startete die Gesundheitsdirektion des Kantons Zürich 2008 das Pilotprojekt «Medical Board», um «einen Beitrag zur Sicherstellung der im Krankenversicherungsgesetz geforderten Wirksamkeit, Zweckmässigkeit und Wirtschaftlichkeit von medizinischen Behandlungen zu leisten». Im September 2009 konnte die Schweizerische Konferenz der Gesundheitsdirektoren (GDK) für das Projekt gewonnen werden. 2010 haben die FMH und die Schweizerische Akademie der Medizinischen Wissenschaften (SAMW) zusammen mit der GDK die Co-Trägerschaft übernommen, und im Fe- bruar 2011 gründeten diese drei Institutionen den Trägerverein Swiss Medical Board (SMB).

Das SMB zeichnete sich durch mutiges Vorgehen, aber auch durch eine gewisse Naivität beim Erstellen seiner Empfehlungen aus. Oft bewirkte dies heftige, teilweise gerechtfertigte Reaktionen. Somit war der finanzielle Support in Frage gestellt. Ausserdem gründeten die Kassen und die Industrie zusammen das SwissHTA mit der Möglichkeit, für sie potentiell nachteilige Entscheide zu kontern.

Zur Gewährleistung der Finanzierung fusionierte das SMB mit dem SwissHTA im Januar 2015. Gegenwärtig wird das SMB von einem neuen Trägerverein bestehend aus GDK, SAMW, dem Fürstentum Liechtenstein, Curafutura, Interpharma und Santésuisse geleitet. Um ihre Unabhängigkeit in Bezug auf HTA-Leistungen zu wahren, ist die FMH (nicht aber die SAMW) aus dem Verein ausgetreten. Zwar nimmt das leitende Organ weder Einfluss auf die Inhalte der Begutachtungs- und Bewertungsleistungen noch auf die Mandatszuweisungen, es entscheidet aber über die strategische Entwicklung, die Definition der operativen Organe, die Auswahl der Themen, die Kommunikation, die Veröffentlichung der Berichte und die Massnahmen zur Umsetzung der jeweiligen Empfehlungen. Die Mitglieder des leitenden Organs - vor allem Industrie und Kassen - können daher die Veröffentlichung von Begutachtungs- und Bewertungsentscheiden blockieren, wenn sie ihnen nicht genehm sind. Durch die Aufgabe der wichtigsten Prinzipien, die solche Strukturen ausmachen - Transparenz, Unabhängigkeit und Glaubwürdigkeit -, hat das SMB daher seine Seele verloren.

Die Unabhängigkeit der HTA-Leistungen kann nur durch staatliche Finanzierung gewährleistet werden, wie dies auch in anderen europäischen Ländern der Fall ist. Eine eidgenössische Finanzierung wurde bereits bei der Zürcher Initiative angeregt, und der Bundesrat scheint nun in dieser Richtung zu agieren. Die systematische und regelmässige Prüfung der Zweckmässigkeit der medizinischen Leistungen ist vorrangiges Ziel der Strategie "Gesundheit2020». Bleibt zu hoffen, dass dieses Vorhaben nicht allein der Einsparung von Gesundheitskosten dienen wird, sondern auch der medizinischen Qualitätsoptimierung, so dass ein neues SMB endlich gemäss anerkannten Kriterien funktionieren kann. 